\title{
Investigating the Accuracy of Quantitative Echocardiographic-Modified Task Force Criteria for Arrhythmogenic Ventricular Cardiomyopathy in Adolescent Male Elite Athletes
}

\author{
Chetanya Sharma ${ }^{1}$ - Dan M. Dorobantu ${ }^{1,2} \cdot$ Diane Ryding $^{4} \cdot$ Dave Perry $^{4} \cdot$ Steven R. McNally ${ }^{4}$ A. Graham Stuart ${ }^{1}$. \\ Craig A. Williams ${ }^{2} \cdot$ Guido E. Pieles $^{1,3,5}$
}

Received: 24 March 2021 / Accepted: 29 September 2021 / Published online: 23 October 2021

(c) The Author(s) 2021

\begin{abstract}
Athlete preparticipation screening focuses on preventing sudden cardiac death (SCD) by detecting diseases such as arrhythmogenic ventricular cardiomyopathy (AVC), which affects primarily the right ventricular myocardium. Diagnosis may be obscured by physiological remodeling of the athlete heart. Healthy athletes may meet the 2010 Task Force Criteria right ventricular outflow tract (RVOT) dimension cut-offs, questioning the suitability of the modified Task Force Criteria (mTFC) in adolescent athletes. In this study, 67 male adolescent footballers undergoing preparticipation screening were reviewed. All athletes underwent a screening for resting ECG and echocardiogram according to the English FA protocol, as well as cardiopulmonary exercise testing, stress ECG, and exercise echocardiography. Athletes' right ventricular outflow tract (RVOT) that met the major AVC diagnostic criteria for dilatation were identified. Of 67 evaluated athletes, 7 had RVOT dilatation that met the major criteria, all in the long axis parasternal view measurement. All had normal right ventricular systolic function, including normal free-wall longitudinal strain (ranging from -21.5 to $-32.7 \%$ ). Left ventricular ejection fraction ranged from 52 to $67 \%$, without evidence of structural changes. Resting ECGs and cardiopulmonary exercise tests were normal in all individuals. In a series of healthy athletes meeting the major AVC diagnostic criteria for RVOT dilatation, none had any other pathological changes on a detailed screening including ECG, exercise testing, and echocardiography. This report highlights that current AVC echocardiographic diagnosis criteria have limitations in this population.
\end{abstract}

Keywords Athletes · Adolescents $\cdot$ Cardiomyopathy $\cdot$ Echocardiography

Guido E. Pieles

guido.pieles@bristol.ac.uk

1 Bristol Congenital Heart Centre, The Bristol Heart Institute, University Hospitals Bristol NHS Foundation Trust, Upper Maudlin Street, Bristol BS2 8BJ, UK

2 Children's Health and Exercise Research Centre, University of Exeter, St Luke's Campus, Heavitree Road, Exeter EX1 2LU, UK

3 National Institute for Health Research (NIHR) Cardiovascular Biomedical Research Centre, Bristol Heart Institute, Upper Maudlin Street, Bristol BS2 8BJ, UK

4 Manchester United Football Club, Football Medicine \& Science Department, AON Training Complex, Birch Road, Carrington, Manchester M31 4BH, UK

5 Institute for Sport Exercise and Health (ISEH), University College Hospital London, London, UK

\section{Introduction}

Arrhythmogenic ventricular cardiomyopathy (AVC, also known as arrhythmogenic right ventricular cardiomyopathy, ARVC) is a rare inherited disease characterized by fibrofatty infiltration of the right ventricular myocardium [1]. Presentation can vary from exercise-induced palpitations to congestive cardiac failure. However, a significant minority present with sudden cardiac death (SCD) is the first symptom [2]. Although the disease usually presents in young adulthood, $10 \%$ of SCDs from AVC occur in children and adolescents [3], and AVC is responsible for at least $20 \%$ of all SCDs in children and adolescents [4]. Athletes with underlying cardiac disease are at greater risk of SCD compared with non-athletic individuals, by virtue of repetitive intense training acting as a trigger for cardiac arrest [5]. There is also a significant male preponderance, with rates in males being 2-25 times higher than females, although the reasons are not 
fully understood $[5,6]$. This increased risk of SCD forms the rationale behind preparticipation screening, as disqualification of affected athletes increases the chance of preventing on-field SCD.

AVC presents a considerable diagnostic challenge as there is no single gold standard method; instead, diagnosis requires a multiparametric approach, as laid out in the modified Task Force Criteria (mTFC) [1] and recently the Padua criteria [7]. In athletes, physiological adaptations to training can obscure and confound the clinical presentation. Ventricular dilatation, mild systolic impairment, and anterior precordial T-wave inversion are well characterized consequences of exercise-induced cardiac remodeling in adults [8-10]. Right ventricular dilatation is a shared characteristic between athletes and AVC. RVOT diameter and RV volume, used in the mTFC and Padua criteria, respectively, may lack specificity in the athlete population. Reference ranges have been proposed for athletes to help differentiate physiological conditioning from AVC but only in adults [11-14]. However, children and adolescents with AVC are less likely to have developed significant structural, functional, and electrical alterations. Both the mTFC and Padua criteria are based almost entirely on non-athletic adult data, and there still remains a lack of pediatric-specific guidance despite acknowledgment that current criteria are inadequate for this group [15]. It has recently been shown that current echocardiographic mTFCs are not met by the majority of adolescent AVC patients [16].

The aim of this study was to assess a group of male adolescent footballers and subsequently report on those with significant right ventricular outflow tract (RVOT) dilatation meeting the size criteria proposed in the MTFC. It was hypothesized that athletes, despite a dilated RVOT, will display no other evidence of cardiac structural or functional abnormality when assessed by conventional 2-dimensional echocardiography, speckle-tracking echocardiography (STE), resting electrocardiogram or cardiopulmonary exercise testing (CPET).

\section{Methods}

Data for male adolescent athletes from an English Premier League football academy undergoing preparticipation screening between 2015 and 2017 were reviewed. All athletes performed more than $10 \mathrm{~h}$ training per week. As part of a prospective case series study, all players signed an assent form, with accompanying parent/guardian consent. National Institute for Health Research (NIHR) ethics board approval was obtained. Participants underwent an evaluation protocol on a single day in the same test facility by two clinicians (GP and CAW). Participants were screened for cardiac disease with a preparticipation questionnaire, physical examination and medical history, 12-lead ECG, resting echocardiography, and exercise echocardiography with cardiopulmonary exercise testing.

An incremental CPET on a recumbent cycle ergometer (Ergosana GMBH, Bitz, Germany) positioned at a $45^{\circ}$ inclination $(25 \mathrm{~W} \cdot 3 / \mathrm{min}$ increments) was performed to volitional exhaustion at a pedaling frequency of $70 \pm 5 \mathrm{rpm}$. Echocardiographic measurements were performed in supine position and analysis performed using an Artida machine, $2.0-4.8 \mathrm{MHz}$ transducer, and UltraExtendV3.2 software (Canon Medical Systems, Japan). Measurements of 2-dimensional, tissue Doppler (TDI) and pulsed-wave (PW) Doppler were recorded in the clinical report and are reproduced in Figs. 1 and 2, for readability only. For the RVOT diameters, one clinician assessed measurements at two different times, and a second clinician (DMD) also assessed the sample. Inter- and intra-observer reliability of RVOT measurements were reported as intraclass coefficient. Speckle-tracking analysis was conducted offline by one clinician (DMD). When warranted, further clinical examinations were recommended based on the local protocol, with no confirmed cardiomyopathy case being reported.

End-diastolic RV diameters were measured in the parasternal long-axis view of the RVOT, proximal parasternal short-axis view of the RVOT, and distal parasternal shortaxis view of the RVOT. Apical views were used to measure the end-diastolic transverse diameter at the RV base $\left(\mathrm{RVD}_{1}\right)$, mid-diameter $\left(\mathrm{RVD}_{2}\right)$, base-to-apex length $\left(\mathrm{RVD}_{3}\right)$, and apical diameter $\left(\mathrm{RVD}_{4)}[16]\right.$. All chamber size measurements were performed in accordance with the current guidelines [17]. RV function was quantified using tricuspid annular peak systolic excursion, RV FAC \%, and PW TDI at the lateral tricuspid annulus [18, 19].

For this case series athletes meeting, the major mTFC criteria for RVOT dilatation (long axis RVOT $\geq 32 \mathrm{~mm}$ or $\geq 19 \mathrm{~mm} / \mathrm{m}^{2}$; short axis proximal RVOT $\geq 36 \mathrm{~mm}$ or $\geq 21 \mathrm{~mm} / \mathrm{m}^{2}$ ) were selected [1]. Right ventricle (RV) volume data were not available at this point, so global RV dilatation Padua Criteria could not be used [7]. RV and LV size, RV function, and LV function age/body size normative values were used to determine abnormal values, in addition to current adult-derived recommendations [17, 20-26]. A left ventricular ejection fraction (LVEF) of less than 55\% was considered abnormal [27].

\section{Results}

Of the 67 athletes evaluated, 7 met the major mTFC for RVOT dilatation (age range 13.4-15.9 years). RVOT sizes for each patient are detailed in Table 1. All 7 had dilated RVOT measured in the parasternal long-axis view $\left(\operatorname{RVOT}_{\text {PLAX }}\right)$, either absolute $(n=5)$ or indexed $(n=2)$, 

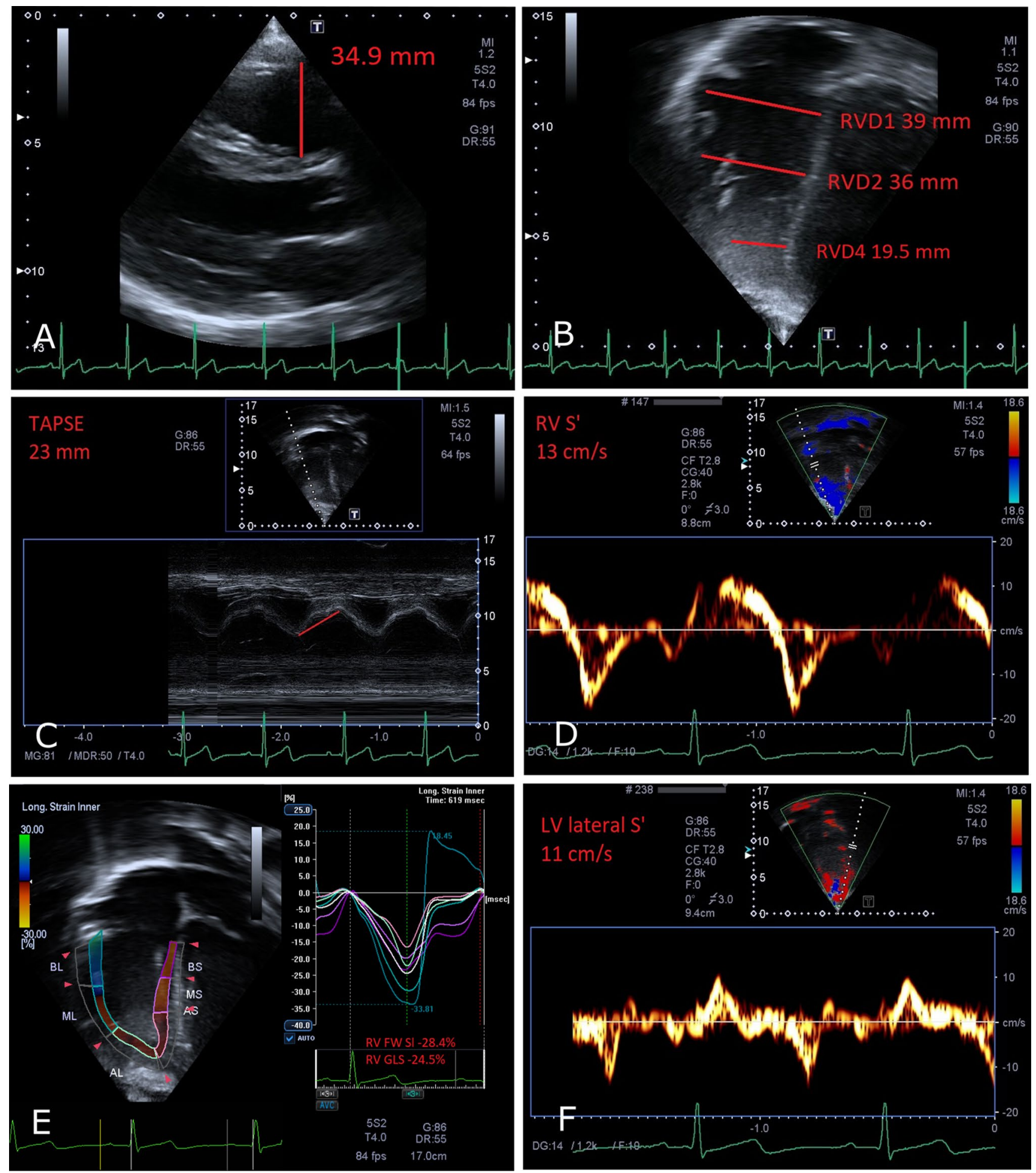

Fig. 1 Echocardiographic biventricular size and systolic function measurements in Athlete 1. A PLAX view showing RVOT end-diastolic diameter. B A4C view showing RV basal $\left(\mathrm{RVD}_{1}\right)$, mid $\left(\mathrm{RVD}_{2}\right)$, and apical $\left(\mathrm{RVD}_{4}\right)$ end-diastolic diameters. $\mathrm{C}$ M-mode view showing TAPSE. D Tissue Doppler imaging showing the peak systolic tricus-

pid annular velocity (RV S'). E Speckle-tracking echocardiography showing RV segmental strain curves, free-wall RV strain (RV Sl), and global RV strain (RV GLS). F Tissue Doppler imaging showing the peak systolic mitral lateral annular velocity (LV lateral S') 

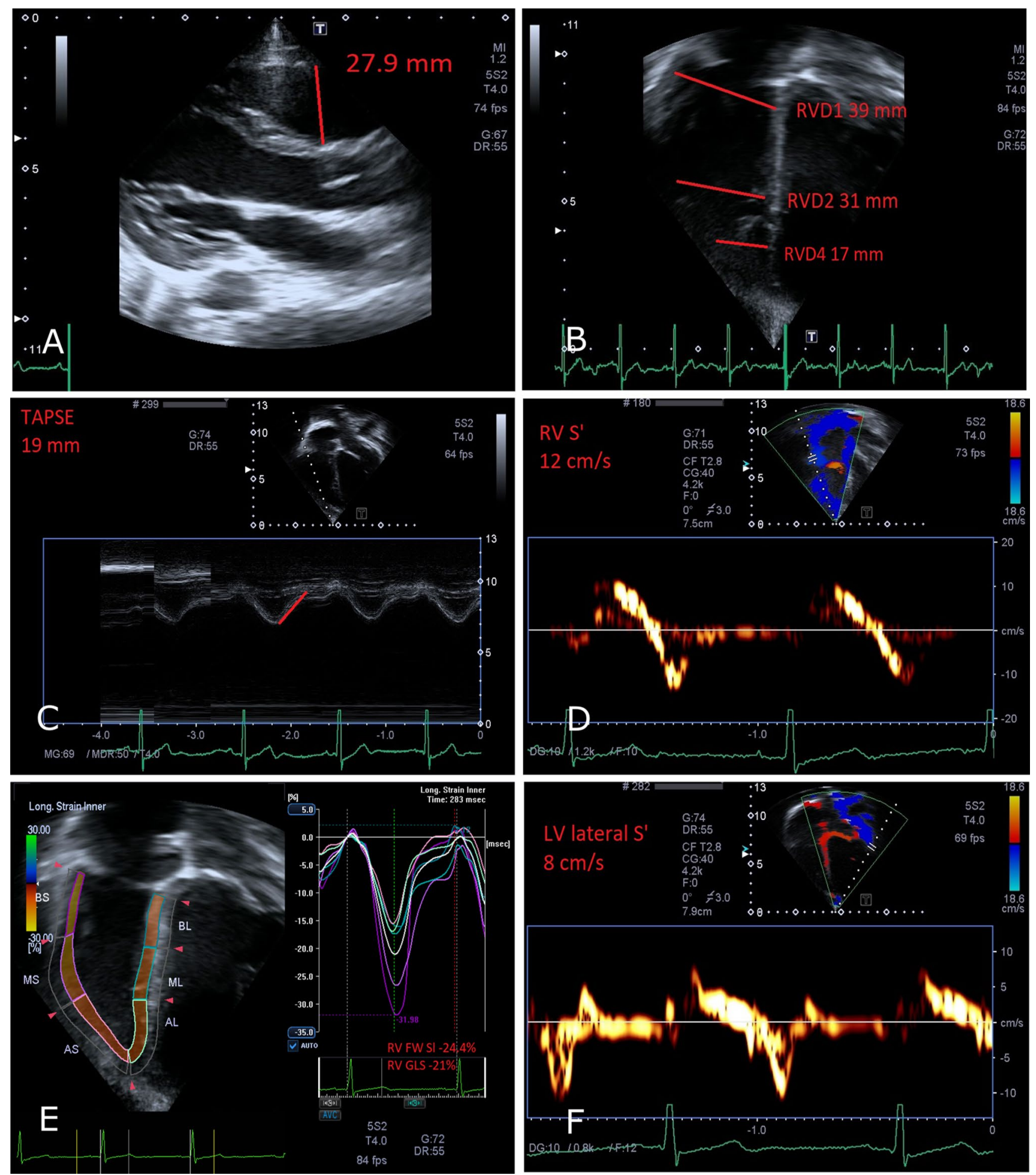

Fig. 2 Echocardiographic biventricular size and systolic function measurements in Athlete 3. Panel descriptions as for Fig. 1

but none met the proximal RVOT dilatation criteria in parasternal short-axis view (RVOT PSAX-proximal $_{\text {) or had }}$ a distal RVOT measured in parasternal short-axis view of $>27 \mathrm{~mm}$. Three athletes had an abnormal/borderline echocardiographic parameter: Athlete 1, Athlete 3, and Athlete 6 . The main echocardiographic findings for athletes 1 and 3 are represented in Figs. 1 and 2, respectively. 
Table 2 details additional RV size and systolic function parameters. One athlete (Athlete 1, Fig. 1) showed a borderline-enlarged $\mathrm{RV}$ mid-inflow diameter $\left(\mathrm{RVD}_{2}\right)$. One athlete (Athlete 6, Fig. 2) showed borderline, but normal for age,
RV peak systolic velocity by TDI. FAC was normal for all athletes. No athletes showed abnormal RV free-wall strain.

The LV size and function parameters are shown in Table 3. Only one athlete (Athlete 3 ) had borderline-low left

Table 1 Individual patient demographics and RVOT measurements

\begin{tabular}{llllllllll}
\hline Participant & Age $(\mathrm{y})$ & Stature $(\mathrm{cm})$ & Body mass $(\mathrm{kg})$ & BSA $\left(\mathrm{m}^{2}\right)$ & $\begin{array}{l}\text { RVOT- } \\
\text { PLAX } \\
(\mathrm{mm})\end{array}$ & $\begin{array}{l}\text { RVOT } \\
\text { BSA }(\mathrm{mm} / \\
\left.\mathrm{m}^{2}\right)\end{array}$ & $\begin{array}{l}\text { RVOT- } \\
\begin{array}{l}\text { PSAX-proximal } \\
(\mathrm{mm})\end{array}\end{array}$ & $\begin{array}{l}\text { RVOT- } \\
\begin{array}{l}\text { PSAX-proximal } \\
\left(\mathrm{mm} / \mathrm{m}^{2}\right)\end{array}\end{array}$ \\
\hline Athlete 1 & 15.9 & 177.4 & 71.7 & 1.9 & 34.9 & 18.5 & 33.1 & 17.6 & $\begin{array}{l}\text { RVOT- } \\
\text { PSAX-distal } \\
(\mathrm{mm})\end{array}$ \\
Athlete 2 & 16.0 & 178.3 & 60.5 & 1.76 & 32.5 & 18.5 & 34.3 & 19.5 & NR \\
Athlete 3 & 14.3 & 157.9 & 44.8 & 1.4 & 27.9 & 19.7 & 26.6 & 18.7 & 22.3 \\
Athlete 4 & 15.2 & 173.8 & 64.2 & 1.8 & 32.3 & 18.2 & 33.3 & 18.8 & 15.8 \\
Athlete 5 & 13.4 & 165.1 & 48.3 & 1.51 & 29.7 & 19.6 & 28.9 & 21.7 \\
Athlete 6 & 15.7 & 179.5 & 74 & 1.93 & 32.7 & 17.0 & 33.2 & 17.2 & 20.3 \\
Athlete 7 & 15.7 & 171.2 & 64.5 & 1.76 & 32.6 & 18.6 & 28.2 & 16 & 22.1 \\
\hline
\end{tabular}

Intra-observer reliability, ICC: RVOT PLAX $=0.73$, RVOT SAX1 $=0.94$, RVOT SAX2 $=0.84$

Inter-observer reliability, ICC: RVOT PLAX $=0.77$, RVOT SAX1 $=0.88$, RVOT SAX2 $=0.60 B S A$ body surface area, $P L A X$ parasternal long axis, $P S A X$ parasternal short axis, RVOT right ventricular outflow tract

Intra-observer reliability, ICC: RVOT PLAX $=0.73$, RVOT SAX1 $=0.94$, RVOT SAX2 $=0.84$

Inter-observer reliability, ICC: RVOT PLAX $=0.77$, RVOT SAX1 $=0.88$, RVOT SAX2 $=0.60$

$B S A$ body surface area, PLAX parasternal long axis, PSAX parasternal short axis, RVOT right ventricular outflow tract

Table 2 Individual participant RV parameters

\begin{tabular}{lllllllll}
\hline Participant & $\mathrm{RVD}_{1}(\mathrm{~mm})$ & $\mathrm{RVD}_{2}(\mathrm{~mm})$ & $\mathrm{RVD}_{3}(\mathrm{~mm})$ & $\mathrm{RVD}_{4}(\mathrm{~mm})$ & $\mathrm{RVS}(\mathrm{cm} / \mathrm{s})$ & TAPSE $(\mathrm{mm})$ & $\begin{array}{c}\text { FAC }(\%) \\
\text { Free wall } \\
\text { strain }(\%)\end{array}$ \\
\hline Athlete 1 & 39 & 36 & 71 & 19.5 & 13 & 23 & 54 & 28.4 \\
Athlete 2 & 37 & 28 & 65 & 18.5 & 15 & 34 & 56 & 32.7 \\
Athlete 3 & 39 & 31 & 67 & 17.0 & 12 & 19 & 45 & 24.4 \\
Athlete 4 & 35 & 25 & 59 & 18.5 & 15 & $\mathrm{NR}$ & 63 & 24.6 \\
Athlete 5 & 42 & 35 & 85 & 19.2 & 14 & 19.9 & 50 & 26.2 \\
Athlete 6 & 40 & 30 & 77 & 19.8 & 13 & 14.3 & 43 & 21.5 \\
Athlete 7 & 39 & 22 & 75 & 22.2 & 12 & 23 & 60 & 27.0 \\
\hline
\end{tabular}

$F A C$ fractional area change, $R V$ right ventricle, $R V D_{1}$ right ventricle basal-inflow diameter, $R V D_{2}$ right ventricle mid-inflow diameter, $R V D_{3}$ right ventricle base-to-apex length, $R V D_{4}$ right ventricle apical-third diameter, $R V S$ ' systolic tricuspid annular velocity, TAPSE tricuspid annular plane systolic excursion

Table 3 Individual LV parameters

\begin{tabular}{lllllllll}
\hline Participant & $\begin{array}{l}\text { IVS } \\
\text { thickness } \\
(\mathrm{cm})\end{array}$ & $\begin{array}{l}\text { PW } \\
\text { thickness } \\
(\mathrm{cm})\end{array}$ & EDD $(\mathrm{cm})$ & ESD $(\mathrm{cm})$ & EF $(\%)$ & LV S' $(\mathrm{cm} / \mathrm{s})$ & E' $(\mathrm{cm} / \mathrm{s})$ & A4C strain \\
\hline Athlete 1 & 1.1 & 0.9 & 4.5 & 3.2 & 61 & 11 & 14 & $\mathrm{NR}$ \\
Athlete 2 & 1.1 & 1.1 & 4.8 & 3.4 & 61 & 11 & 17 & 19.86 \\
Athlete 3 & 0.9 & 0.8 & 4.3 & 3.2 & 52 & 8 & 10 & $\mathrm{NR}$ \\
Athlete 4 & 0.9 & 1.1 & 4.3 & 3.0 & 60 & $\mathrm{NR}$ & $\mathrm{NR}$ & $\mathrm{NR}$ \\
Athlete 5 & 1 & 0.7 & 3.9 & 2.4 & 59 & 10 & 16 & 20.26 \\
Athlete 6 & 1.1 & 1 & 4.6 & 3.3 & 67 & 11.5 & 12 & $\mathrm{NR}$ \\
Athlete 7 & 1.1 & 1 & 4.9 & 3.9 & 56 & 10 & 17 & 19.32 \\
\hline
\end{tabular}

$A 4 C$ apical-4-chamber, $E D D$ end-diastolic diameter, $E F$ ejection fraction, $E S D$ end-systolic diameter, IVS interventricular septum, $L V$ left ventricle, $N R$ not recorded, $P W$ posterior wall 
ventricular ejection fraction and $\mathrm{LV}$ peak systolic velocity by TDI. All other LV parameters, including apical-4-chamber strain, were within the normal range for all athletes.

All athletes had a normal resting 12-lead ECG, normal cardiopulmonary exercise test (CPET) values of a trained athlete and normal exercise echocardiogram.

\section{Discussion}

This study assessed reported detailed clinical, imaging, and physiological data on adolescent athletes with significant right ventricular outflow tract (RVOT) dilatation meeting the 2010 mTFC [1]. There were no significant echocardiographic (including speckle tracking), ECG, CPET, or exercise echocardiographic findings suggestive of pathology, except for the RVOT dilation. Athletes had no relevant symptomatology, family history, or past medical history. These observations show the need to re-evaluate how RVOT dilatation is interpreted for the diagnosis of AVC in adolescent athletes. At present, there is a paucity of research on the subject of using the mTFC for AVC in the pediatric athlete population, against a background of increasing participation of children and adolescents in competitive sport [28]. In this unique patient population, two challenges in diagnosis arise: differentiating pathology from physiology of the athlete heart and the need to use criteria designed for adults in a pediatric setting.

In our sample of 67 athletes, 7 athletes met the struc-

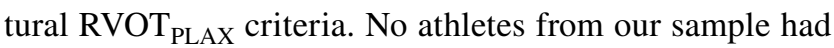

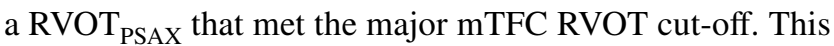
is in-keeping with a systematic review and meta-analysis by D'Ascenzi et al. (2017) on normative right heart values in adult athletes, which found that male athletes had higher upper limits for $\mathrm{RVOT}_{\text {PLAX }}$, while $\mathrm{RVOT}_{\mathrm{PSAX}}$ values were comparable to controls [13].

It is important to note that while the included athletes fulfilled the quantitative major echocardiographic thresholds of the mTFC, they did not display the prerequisite regional $\mathrm{RV}$ akinesia, dyskinesia, or aneurysm required for the mTFC echocardiographic major criterion. However, in practice, clinicians cannot rely on these morphological abnormalities given that most children would be expected to be in the early stages of disease. Furthermore, visual assessment of these functional abnormalities may be challenging in young athletes exhibiting significant RV remodeling [29]. In comparison with cardiac magnetic resonance imaging (CMR), echocardiography may be insensitive to early and subtle changes due to limitations in acoustic windows to the RV free wall [30]. Indeed, one study showed that only $50 \%$ of patients with CMR-positive AVC fulfilled echocardiographic mTFC [31]. Therefore, clinicians should not expect adolescents to exhibit the RV wall motion abnormalities that are the hallmark of AVC, representing a further limitation of both the mTFC and the Padua criteria in adolescents. Crucially, this leads to an inevitable increased reliance on RVOT dimensions to guide any suspicion of diagnosis during preparticipation screening, which, as shown in this study, are not appropriate in the adolescent athlete population and might lead to unnecessary investigations, delays in treatment, and patient anxiety. In addition, while diagnostic criteria encourage scaling of RVOT dimensions to body surface area (BSA), this is not always helpful in differentiating AVC from controls [16] and allometric scaling of RV chamber size to BSA may better ensure size independence [11].

Novel techniques such as speckle-tracking echocardiography have been shown to be useful discriminators in adults and adolescents with AVC and help in the early diagnosis $[16,32]$. In a recent study, RV longitudinal strain and the apical RV end-diastolic diameter $\left(\mathrm{RVD}_{4}\right)$, which was, therefore, also included in our analysis (Table 2), were abnormal in adolescent AVC patients and predictors of disease, despite not meeting the mTFC for RVOT dilatation [16]. These data also support our finding that the mTFCs have a low diagnostic sensitivity in adolescents. Furthermore, using RV strain measurements may not only increase the diagnostic yield of echocardiography in AVC to diagnose but also exclude the disease, as shown in our cohort.

The recently proposed Padua criteria replace RVOT diameter with end-diastolic volumes in the morpho-functional category for AVC diagnosis. While these useful parameters are readily obtained by modern CMR, they are challenging to obtain using echocardiography and, while possible, are not part of standard assessment [33]. As in the mTFC, there persists the prerequisite for RV akinesia, dyskinesia, or bulging, with its aforementioned limitations in the pediatric population as they are rarely found using echocardiography in adolescents with AVC [16, 30].

The limitations of this case study design are acknowledged. No statistical comparisons were made with non-athletic adolescents or the adolescent athletes not meeting the mTFC for RVOT dilatation. Instead, this study sought to explore individual case data rather than aggregate results, so that granular, detailed data on each athlete could be presented. We also did not explore other mTFC parameters such as cardiac magnetic resonance imaging, family history, and genetics. Furthermore, only male football players were included, thus, not representing a truly diverse athlete population with female players and other sporting disciplines.

In the future, new techniques such as tissue Doppler and speckle-tracking imaging should be explored in preparticipation screening, especially in cases of abnormal structural changes such as RVOT dilatation. Challenges in implementing these techniques include a lack of data-acquisition protocols and normative data for adolescent athletes, as well as operator experience with STE analysis. Furthermore, routine 
preparticipation echocardiography does imply an increase on resource allocation, from experienced clinical staff to increased costs, and for these reasons, its implementation is still sparse.

In summary, it was shown in this adolescent athlete population that a dilated RVOT in isolation should not be viewed as a 'red flag' for the diagnosis of AVC. The results from this case series indicate the need to re-evaluate the appropriateness of current echocardiographic criteria for AVC diagnosis in adolescent athletes. While the limitations of echocardiography in the diagnosis of AVC are acknowledged, echocardiography is the primary imaging modality in cardiac screening of athletes, and therefore, more accurate screening protocols for AVC in this population are needed.

Funding This study was supported by the National Institute for Health Research (NIHR) Biomedical Research Centre at the University Hospitals Bristol NHS Foundation Trust and the University of Bristol. The views expressed in this publication are those of the author(s) and not necessarily those of the NHS, the National Institute for Health Research or the Department of Health. At the time of the study, GEP held a NIHR Academic Clinical Lectureship. DMD is supported by a doctoral scholarship (grant MR/N0137941/1 for the GW4 BIOMED DTP, awarded to the Universities of Bath, Bristol, Cardiff, and Exeter from the Medical Research Council (MRC)/UKRI). We would like to thank the athletes and staff at Manchester United Football Club Youth Academy for their commitment to this research project. This study is part of a research partnership between Canon Medical Systems UK, Manchester United Football Club and the Universities of Bristol and Exeter. The research partnership between Canon Medical Systems and the University of Bristol is a contractual research partnership that determines the independence of the research from either parties' interests.

\section{Declarations}

Conflict of interest The authors declare that they have no conflict of interest.

Ethical Approval National Institute for Health Research (NIHR) ethics board approval was obtained.

Open Access This article is licensed under a Creative Commons Attribution 4.0 International License, which permits use, sharing, adaptation, distribution and reproduction in any medium or format, as long as you give appropriate credit to the original author(s) and the source, provide a link to the Creative Commons licence, and indicate if changes were made. The images or other third party material in this article are included in the article's Creative Commons licence, unless indicated otherwise in a credit line to the material. If material is not included in the article's Creative Commons licence and your intended use is not permitted by statutory regulation or exceeds the permitted use, you will need to obtain permission directly from the copyright holder. To view a copy of this licence, visit http://creativecommons.org/licenses/by/4.0/.

\section{References}

1. Marcus FI, McKenna WJ, Sherrill D, Basso C, Bauce B, Bluemke DA et al (2010) Diagnosis of arrhythmogenic right ventricular cardiomyopathy/dysplasia: proposed modification of the task force criteria. Circulation 121(13):1533-1541

2. Kies PBM, Bax J, Schalij MJ, van der Wall EE (2006) Arrhythmogenic right ventricular dysplasia/cardiomyopathy: screening, diagnosis, and treatment. Heart Rhythm 3:225-234

3. Tabib A, Loire R, Chalabreysse L, Meyronnet D, Miras A, Malicier D et al (2003) Circumstances of death and gross and microscopic observations in a series of 200 cases of sudden death associated with arrhythmogenic right ventricular cardiomyopathy and/ or dysplasia. Circulation 108(24):3000-3005

4. Pilmer CM, Kirsh JA, Hildebrandt D, Krahn AD, Gow RM (2014) Sudden cardiac death in children and adolescents between 1 and 19 years of age. Heart Rhythm 11(2):239-245

5. Corrado D, Basso C, Rizzoli G, Schiavon M, Thiene G (2003) Does sports activity enhance the risk of sudden death in adolescents and young adults? J Am Coll Cardiol 42(11):1959-1963

6. Harmon KG, Asif IM, Maleszewski JJ, Owens DS, Prutkin JM, Salerno JC et al (2015) Incidence, cause, and comparative frequency of sudden cardiac death in national collegiate athletic association athletes: a decade in review. Circulation 132(1):10-19

7. Corrado D, Perazzolo Marra M, Zorzi A, Beffagna G, Cipriani A, Lazzari M et al (2020) Diagnosis of arrhythmogenic cardiomyopathy: the Padua criteria. Int J Cardiol. https://doi.org/10.1016/j. ijcard.2020.06.005

8. Zaidi A, Ghani S, Sharma R, Oxborough D, Panoulas VF, Sheikh $\mathrm{N}$ et al (2013) Physiological right ventricular adaptation in elite athletes of African and Afro-Caribbean origin. Circulation 127(17):1783-1792

9. Henriksen EKT, Jonason T, Landelius J, Hedberg P, Ekstrand P, Ringqvist I (1998) An echocardiographic study of right ventricular adaptation to physical exercise in elite male orienteers. Clin Physiol 18:498-503

10. Ector J, Ganame J, van der Merwe N, Adriaenssens B, Pison L, Willems R et al (2007) Reduced right ventricular ejection fraction in endurance athletes presenting with ventricular arrhythmias: a quantitative angiographic assessment. Eur Heart J 28(3):345-353

11. Oxborough D, Sharma S, Shave R, Whyte G, Birch K, Artis $\mathrm{N}$ et al (2012) The right ventricle of the endurance athlete: the relationship between morphology and deformation. J Am Soc Echocardiogr 25(3):263-271

12. D'Ascenzi F, Anselmi F, Piu P, Fiorentini C, Carbone SF, Volterrani $L$ et al (2019) Cardiac magnetic resonance normal reference values of biventricular size and function in male athlete's heart. JACC Cardiovasc Imaging 12(9):1755-1765

13. D'Ascenzi F, Pelliccia A, Solari M, Piu P, Loiacono F, Anselmi F et al (2017) Normative reference values of right heart in competitive athletes: a systematic review and meta-analysis. J Am Soc Echocardiogr 30(9):845-58 e2

14. Hoorntje ET, Te Rijdt WP, James CA, Pilichou K, Basso C, Judge DP et al (2017) Arrhythmogenic cardiomyopathy: pathology, genetics, and concepts in pathogenesis. Cardiovasc Res 113(12):1521-1531

15. Corrado D, van Tintelen PJ, McKenna WJ, Hauer RNW, Anastastakis A, Asimaki A et al (2020) Arrhythmogenic right ventricular cardiomyopathy: evaluation of the current diagnostic criteria and differential diagnosis. Eur Heart J 41(14):1414-1429

16. Pieles GE, Grosse-Wortmann L, Hader M, Fatah M, Chungsomprasong P, Slorach C et al (2019) Association of echocardiographic parameters of right ventricular remodeling and myocardial performance with modified task force criteria in adolescents with 
arrhythmogenic right ventricular cardiomyopathy. Circ Cardiovasc Imaging 12(4):e007693

17. Lang RM, Badano LP, Mor-Avi V, Afilalo J, Armstrong A, Ernande L et al (2015) Recommendations for cardiac chamber quantification by echocardiography in adults: an update from the American society of echocardiography and the European association of cardiovascular imaging. J Am Soc Echocardiogr 28(1):139 e14

18. Mertens LL, Friedberg MK (2010) Imaging the right ventriclecurrent state of the art. Nat Rev Cardiol 7(10):551-563

19. Lopez L, Colan SD, Frommelt PC, Ensing GJ, Kendall K, Younoszai AK et al (2010) Recommendations for quantification methods during the performance of a pediatric echocardiogram: a report from the pediatric measurements writing group of the American society of echocardiography pediatric and congenital heart disease council. J Am Soc Echocardiogr 23(5):465-95 (quiz 576-7)

20. Cantinotti M, Scalese M, Murzi B, Assanta N, Spadoni I, De Lucia $V$ et al (2014) Echocardiographic nomograms for chamber diameters and areas in Caucasian children. J Am Soc Echocardiogr. 27(12):1279-922

21. Koestenberger M, Ravekes W, Everett AD, Stueger HP, Heinzl B, Gamillscheg A et al (2009) Right ventricular function in infants, children and adolescents: reference values of the tricuspid annular plane systolic excursion (TAPSE) in 640 healthy patients and calculation of z score values. J Am Soc Echocardiogr 22(6):715-719

22. Koestenberger M, Nagel B, Ravekes W, Avian A, Heinzl B, Cvirn $G$ et al (2012) Reference values of tricuspid annular peak systolic velocity in healthy pediatric patients, calculation of $\mathrm{z}$ score, and comparison to tricuspid annular plane systolic excursion. Am J Cardiol 109(1):116-121

23. Moiduddin N, Texter KM, Zaidi AN, Hershenson JA, Stefaniak CA, Hayes J et al (2010) Two-dimensional speckle strain and dyssynchrony in single right ventricles versus normal right ventricles. J Am Soc Echocardiogr 23(6):673-679

24. Cantinotti M, Scalese M, Giordano R, Franchi E, Assanta N, Marotta $\mathrm{M}$ et al (2018) Normative data for left and right ventricular systolic strain in healthy Caucasian Italian children by two-dimensional speckle-tracking echocardiography. J Am Soc Echocardiogr. 31(6):712-206

25. van der Hulst AE, Delgado V, Ten Harkel AD, Klitsie LM, Filippini LH, Bax JJ et al (2011) Tissue Doppler imaging in the left ventricle and right ventricle in healthy children: normal age-related peak systolic velocities, timings, and time differences. Eur J Echocardiogr 12(12):953-960

26. Lemmer CE, Engel ME, Stanfliet JC, Mayosi BM (2014) Reference intervals for the echocardiographic measurements of the right heart in children and adolescents: a systematic review. Cardiovasc Ultrasound. https://doi.org/10.1186/1476-7120-12-3

27. Harkness A, Ring L, Augustine DX, Oxborough D, Robinson S, Sharma V (2020) Normal reference intervals for cardiac dimensions and function for use in echocardiographic practice: a guideline from the British society of echocardiography. Echo Res Pract 7(1):G1-G18

28. Caine D, Maffulli N, Caine C (2008) Epidemiology of injury in child and adolescent sports: injury rates, risk factors, and prevention. Clin Sports Med 27(1):19-50 vii

29. D’Ascenzi F, Pelliccia A, Valentini F, Malandrino A, Natali BM, Barbati $R$ et al (2017) Training-induced right ventricular remodelling in pre-adolescent endurance athletes: the athlete's heart in children. Int J Cardiol 236:270-275

30. Etoom Y, Govindapillai S, Hamilton R, Manlhiot C, Yoo S-J, Farhan $M$ et al (2015) Importance of CMR within the task force criteria for the diagnosis of ARVC in children and adolescents. J Am Coll Cardiol 65(10):987-995

31. Borgquist R, Haugaa KH, Gilljam T, Bundgaard H, Hansen J, Eschen $\mathrm{O}$ et al (2014) The diagnostic performance of imaging methods in ARVC using the 2010 task force criteria. Eur Heart J Cardiovasc Imaging 15(11):1219-1225

32. Teske AJ, Cox MG, De Boeck BW, Doevendans PA, Hauer RN, Cramer MJ (2009) Echocardiographic tissue deformation imaging quantifies abnormal regional right ventricular function in arrhythmogenic right ventricular dysplasia/cardiomyopathy. J Am Soc Echocardiogr 22(8):920-927

33. Ostenfeld E, Flachskampf FA (2015) Assessment of right ventricular volumes and ejection fraction by echocardiography: from geometric approximations to realistic shapes. Echo Res Pract 2(1):R1-R11

Publisher's Note Springer Nature remains neutral with regard to jurisdictional claims in published maps and institutional affiliations. 